\title{
Magnetic resonance evaluation of acute abdominal and pelvic pain in pregnancy
}

\author{
Yasser Hassan Habash ${ }^{1}$ and Hoda Salah Darwish ${ }^{2 *}$ \\ *Correspondence: darwish.hoda@yahoo.com \\ 'Lecturer of Obstetrics and Gynecology, Faculty of Medicine, Suez Canal University, Ismailia, Egypt. \\ ${ }^{2}$ Lecturer of Radiodiagnosis, Faculty of Medicine, Suez Canal University, Ismalia, Egypt.
}

\begin{abstract}
Purpose: The aim of this study was to evaluate the efficacy of magnetic resonance (MR) in the assessment of pregnant women with acute abdominal and pelvic pain.

Patients and methods: A prospective study was conducted from April 2011 to March 2013 in Security Forces Hospital, Riyadh, Kingdom of Saudi Arabia (K.S.A). MRI study of 25 patients presented with acute abdominal and/or pelvic pain in which ultrasound was indeterminate, were included in this study. Multiplanar magnetic resonance images of the pelvis were obtained and independently reviewed by two radiologists with discrepancies was resolved by consensus. Data from both magnetic resonance imaging and ultrasonography were obtained, and the definite diagnosis was established with laparoscopic or open surgical findings and results of clinical follow-up as the reference standard.

$\underline{\text { Results: }}$ Correlation of prospective clinical MR interpretations with follow-up medical records showed correct identification of disease entities in all patients. The following disease processes were identified using MRI: appendicitis $(n=3)$, pyelonephritis $(n=3)$, hydronephrosis $(n=2)$, pancreatitis $(n=2)$, uterine fibroid degeneration $(n=4)$, degeneration and torsion of a subserosal uterine fibroid $(n=2)$, simple ovarian cysts $(n=3)$, and ovarian torsion $(n=2)$, ulcerative colitis $(n=1)$, Crohn's disease with perianal fistula $(n=1)$, and pelvic congestion syndrome $(n=1)$. Two of the 25 patients had normal findings on MR examinations and unremarkable follow-up.

Conclusion: Magnetic resonance imaging (MRI) is an accurate, useful and safe in detecting the cause of acute abdominal/pelvic pain in pregnant patients.
\end{abstract}

Keywords: Acute pelvic pain, ultrasonography, magnetic resonance imaging, abdominal pain

\section{Introduction}

The term "acute pain" was defined as pain that was sudden in onset and had been present for $72 \mathrm{hr}$ or less [1].

Acute abdominal pain in pregnant patients presents a difficult diagnostic challenge. The differential diagnosis during pregnancy is extensive in that the abdominal pain may be obstetric in nature or may be caused by disease of other intraabdominal or intra-pelvic structures [2]. It is associated with a long differential diagnosis, and diagnostic imaging is often required to guide management [3].

Because of the anatomic and physiologic changes that occur with pregnancy, localization of disease can be difficult [1].

Computed tomography provides excellent anatomic detail, but exposes patients to ionizing radiation, which can be problematic, as it is conferred to the fetus, making this technique undesirable [1-4].

Sonography is a safe, versatile imaging technique to use in pregnant patients and is often the first imaging technique used in a pregnant patient with abdominal pain. However, in the presence of a gravid uterus, intra-abdominal organs may be displaced and challenging to visualize on ultrasonography, and it might be inconclusive, even when combined with color and pulsed Doppler images [4]. Also, the maternal structures may be difficult to evaluate during pregnancy due to anatomic changes [3].

With its high contrast resolution, its ability to provide good tissue characterization, and its multiplanar imaging capabilities, magnetic resonance imaging (MRI) is increasingly used to evaluate pelvic pathology [5-6]. This technique has superb soft-tissue contrast, particularly of the gynecologic organs, additional benefits include absence of ionizing radiation and exposure to iodinated contrast material that making it safe to use in pregnant [7].

The aim of our study was to evaluate the efficacy of magnetic resonance (MR) in the assessment of acute abdomen/pelvic pain in pregnant patients.

\section{Patients and methods}

Patients presented to the obstetric emergency room of Security Forces Hospital, Kingdom of Saudi Arabia (K.S.A) during the period from April 2011 to March 2013 with acute pelvic pain associated with or without other symptoms like nausea, vomiting, vaginal bleeding, or fever $(n=25)$ were included in our study.

The informed consent was obtained from each subject or subject's guardian, also the institutional review board approval was obtained from Security Forces Hospital Institutional review 
Habash et al. Medical Imaging and Radiology 2013,

board (IRB).

All patients were subjected to medical history and clinical examination, pelvic ultrasound was the initial screening investigation done for all patients, and MRI. Fetal sonographic examination also was before MRI study for all patients. Ultrasound pelvis was done using ultrasound machine (HD II XE Ultrasound 2006, Philips medical system, Netherlands B.V). A curved array transducer (C5-2, model 21426 A) for transabdominal applications was used for all the patients. All patients underwent MRI pelvis using MRI scanner (Optima ${ }^{T M}$ MR $450 \mathrm{~W} 1.5$ Tesla). MRI of the pelvis consisted of the following sequences: three-plane localizer; axial, sagittal and coronal T1-weighted fast spin echo (FSE), axial T2-weighted fast recovery fast spin echo (FRFSE) fat sat. Coronal, and sagittal T2weighted FRFSE, axial, sagittal and coronal short time inversion recovery (STIR), axial T1-weighted fat-suppressed, axial diffusion-weighted images $\left(b=0, b=400 \& b=800 \mathrm{sec} / \mathrm{mm}^{2}\right)$ with fat saturation.

Post contrast axial, coronal and sagittal T1-weighted images with fat saturation were obtained after giving the patient a dose of $0.1 \mathrm{mmol} / \mathrm{kg}$ of Gadolinium that was injected automatically at a rate of $2 \mathrm{ml} / \mathrm{s}$.

The nature of the MRI examination and its associated risks and benefits were explained to all patients before the study, and signed consent was obtained from all patients.

Medical, surgical, and obstetric records were reviewed to determine the correctness of the clinical MR interpretations.

\section{Statistical analysis}

Data was analyzed using commercially available software (statistical package for social sciences, SPSS version 17).

\section{Results}

A total of 25 patients were included in our study, the patient age range was $18-37$ years (mean age 24.5 years $+/-6.5$ years SD) and the mean gestational age was 23 weeks (range, 12-35 weeks).

The following disease processes were identified using MRI: appendicitis $(n=3)$, pyelonephritis $(n=3)$, hydronephrosis $(n=2)$, pancreatitis $(n=2)$, uterine fibroid degeneration $(n=4)$, degeneration and torsion of a subserosal uterine fibroid $(n=2)$, simple ovarian cysts $(n=3)$, and ovarian torsion $(n=2)$, ulcerative colitis $(n=1)$, Crohn's disease with perianal fistula $(n=1)$, and pelvic congestion syndrome $(n=1)$.

Two of the 25 patients had normal findings on MR examinations and unremarkable follow-up.

In our study, three patients with suspected appendicitis, sonographic examination did not depict the appendix. Ultrasound of one patient revealed minimal free pelvic collection at right iliac fossa. Subsequent MR study was done, in the first 2 patients, MRI revealed acute inflammation of the appendix with minimal peri- appendiceal fat stranding. In the third patient MRI study revealed mild peri-appendiceal fluid collection as well as enlarged appendix (Figure 1). An

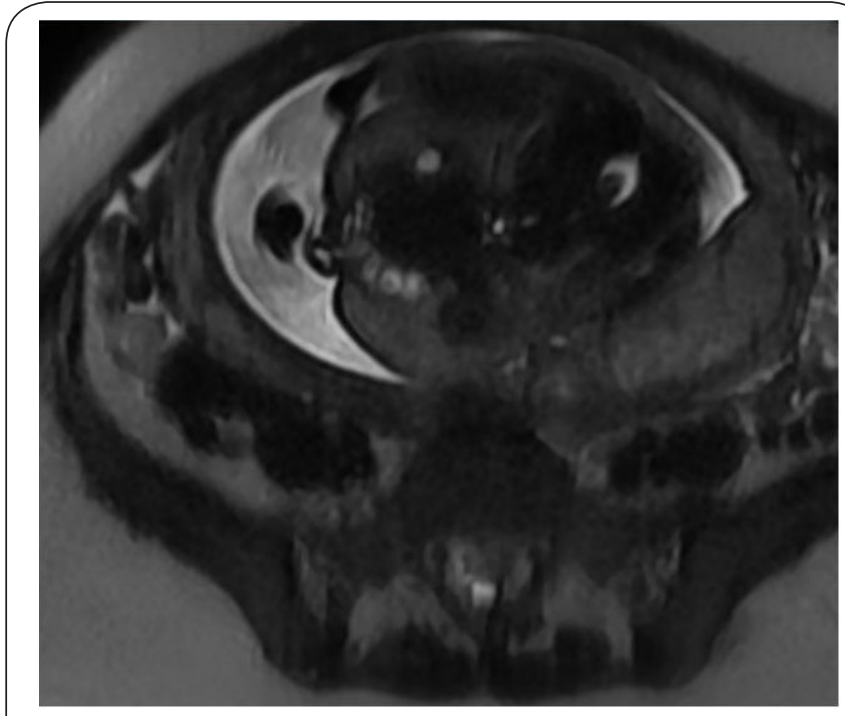

Figure 1. Acute appendicitis: 25 years old pregnant of 36 weeks gestation presented with acute pelvic pain mainly at right iliac fossa associated with fever. Axial fat suppressed T2WI showing enlarged appendix with mild periappendicular fluid in keeping with acute appendicitis.

open appendectomy was done for all our 3 patients. Post operative changes was noted with MRI study in one of these 3 patients (Figure 2).

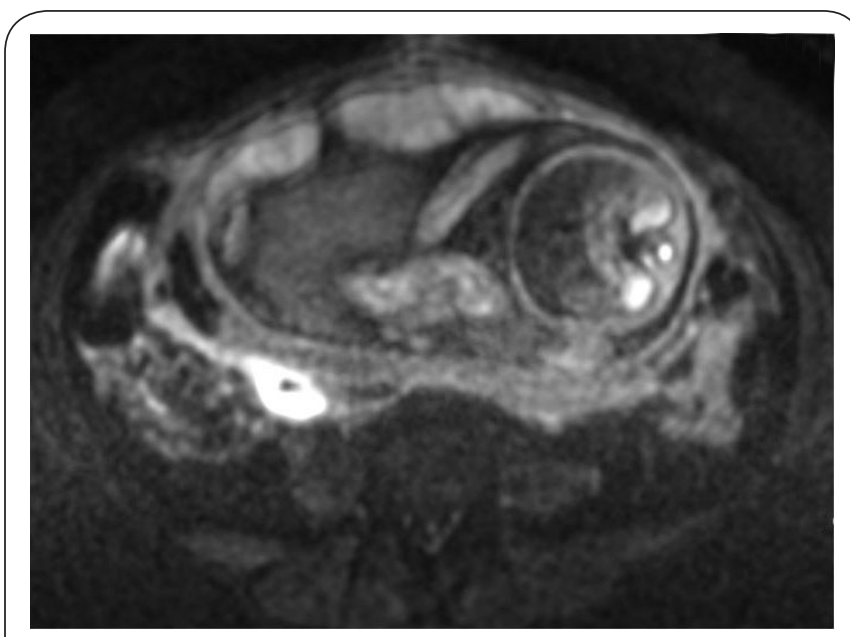

Figure 2. Post operative changes: Axial diffusion weighted images showing an area of diffusion restriction at the expected sit of the appendix right postero-lateral to the gravid uterus attributed to post operative changes.

In our study 2 patients showing moderate hydronephrosis one of them had distal right ureteric stone causing acute ureteric obstruction.

In our study 4 patients showing uterine fibroid degeneration, another 2 patients with acute pelvic pain at 29 and 31 weeks gestation respectively, MRI showed degeneration and 
suspected torsion of a subserosal uterine fibroid in both patients. Ultrasonography revealed a parauterine mass without Doppler flow but did not show the origin of the mass in both patients while MRI study showed a clear connection of the mass to the uterus by a narrow pedicle and normal ovaries in first patient while in the second patients long pedicle is noted.

Laparotomy was done in both patients and revealed a torsed subserosal fibroid rotated $360^{\circ}$ on a very narrow stalk in one patients and long stalk in the second one. In our study we have 3 cases diagnosed as simple ovarian cyst, US showing simple ovarian cyst, (Figure 3). Doppler study was inconclusive in the 3 cases regarding the diagnosis of torsion as it revealed minimal peripheral flow. MRI study excluded the diagnosis of torsion (Figure 4). Lapraotomy was done for the 3 cases after delivery.

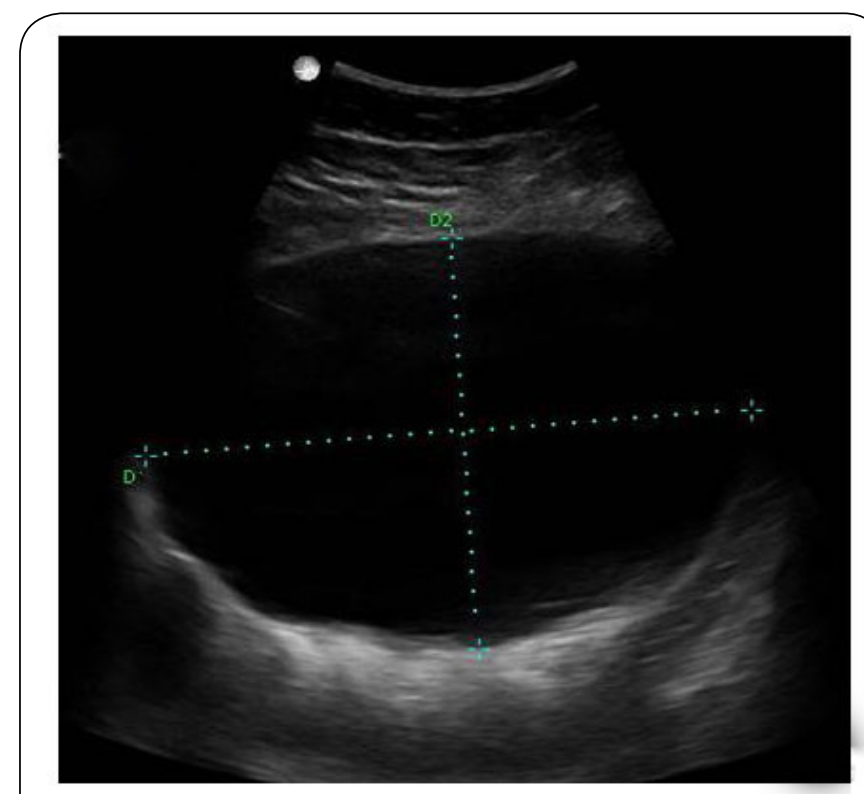

Figure 3. Simple right ovarian cyst: 26 years old female presented with right sided acute pelvic pain, patient was pregnant 32 weeks gestation, Gray scale trans-abdominal US showing a large well defined right adnexal cystic lesion, measures 13.2x 10.3x $8.5 \mathrm{~cm}$ with clear content. Color Doppler shows perilesional minimal blood flow.

In our study 2 cases diagnosed as inflammatory bowel disease, one was ulcerative colitis and the other one Crohn's disease with perianal fistula, in both cases, colonic biopsy confirmed the diagnoses. The patient delivered at 34 and 35 weeks respectively because of the premature rupture of membranes. One of our patients showing with engorged bilateral parametrial venous plexus and ovarian veins with MRI study done 5 days after appendectomy suggestive of pelvic congestion syndrome and ovarian vein syndrome (Figures $5 a$ and $5 b$ ).

The latter three cases ( 2 cases inflammatory bowel disease and one case pelvic congestion syndrome) were inconclusive

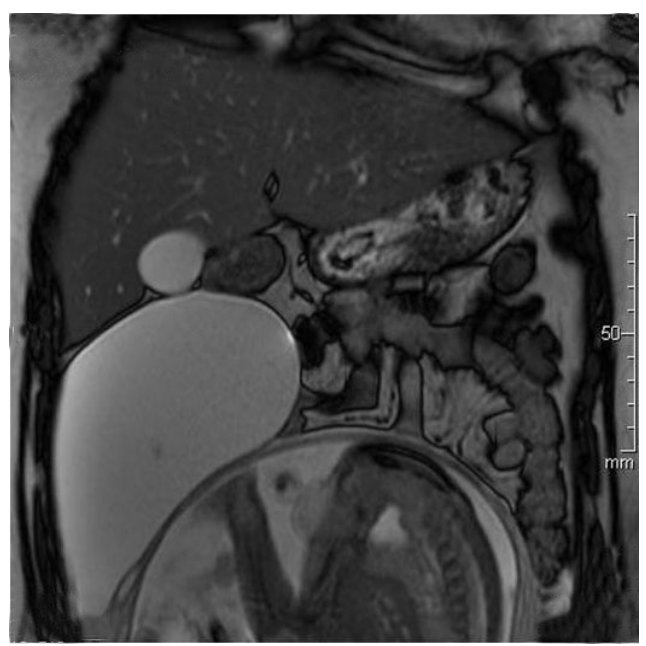

Figure 4. T2 coronal reformatted images of same patient: revealed a large well defined oval shaped thin walled cyst seen at right mid abdomen between the gravid uterus and the liver. It displays low signal intensity on T1WI and high signal intensity on T2WI. No internal hemorrhage or soft tissue component. No signs of inflammation or torsion.
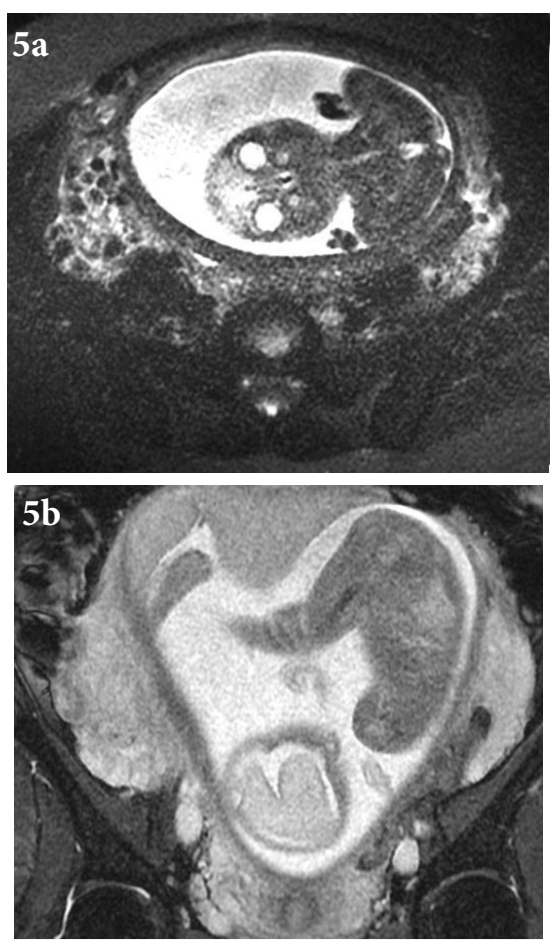

Figure 5. Pelvic congestion syndrome (a) Axial DWI FSE: 29 years old pregnant lady, gestational age of 26 weeks, day 5 after laparascopic appendectomy complaining of acute pelvic. (b) Coronal 2D Fiesta showing showing engorged parametrium venous plexus at both sides as well as ovarian veins highly suggestive of pelvic congestion syndrome and ovarian vein syndrome. 
Habash et al. Medical Imaging and Radiology 2013,

by US and were diagnosed by MRI.

\section{Discussion}

The pregnant patient with acute abdominal or pelvic pain presents a unique diagnostic challenge [1]. One of the challenges facing clinicians is the wide range of differential diagnoses that must be considered when assessing abdominal pain. In conjunction with clinical findings, various imaging modalities play an important role in diagnosing the cause of pain. In patients presenting with acute pain ultrasound is usually employed as the first imaging modality as it is highly sensitive, fast and easy to access. MRI is not usually used in the acute setting but it becomes an important tool in characterization and final diagnosis of abnormalities that remain indeterminate following ultrasound examination [8].

Results of our study show the ability of MRI to accurately characterize pelvic disease in pregnant patients with acute abdominal/pelvic pain. MRI has the ability to evaluate abdominal and pelvic disease [9]. It has been shown to be a useful imaging technique in the diagnosis of appendicitis [10], inflammatory bowel disease [11], pancreatitis, intussusception [1], hydronephrosis and pyelonephritis, uterine fibroids, and adnexal masses [12-13].

The diagnosis of appendicitis is commonly missed in pregnant patients because of many reasons includes: leukocytosis occurs physiologically during pregnancy; nausea and emesis are common symptoms of appendicitis but are common during pregnancy [14] besides that the anatomic alterations in the location of the appendix that accompany an enlarging uterus [1].

Pyelonephritis is important to be excluded in pregnant patients as it associated with premature labor [1].

Hydronephrosis and hydroureter occur in $70-90 \%$ of pregnancies because of mechanical obstruction from an enlarged uterus or diminished muscle tone in the urinary tract from hormonal changes associated with pregnancy and patients may develop ureteral obstruction and require ureteral stents or nephrostomy tubes in sever cases [15].

Presentation of pancreatitis in pregnancy is similar to that in non-pregnant patients. Its causes include biliary sludge and gallstones, which are the most common cause of pancreatitis during pregnancy [1-16].

The diagnosis of pancreatitis is usually clinical, however MRI is useful for characterizing complicated pancreatitis. Maternal mortality is low in uncomplicated pancreatitis, but it is greater than $10 \%$ in complicated pancreatitis [1].

Fibroids (leiomyoma) are the most common pelvic tumors affecting females in the fertile age group. They occur in $20-40 \%$ of females above 30 years of age [17]. As fibroids may cause acute pain, patients may present at the emergency department. Acute pain may be caused by the degeneration of a fibroid when it outgrows its blood supply, torsion of a pedunculated fibroid or prolapse of a submucosal fibroid [9].

Uterine fibroids tend to become smaller during pregnancy, and some involutes completely. However, large fibroids can undergo hemorrhagic infarction, which results in pain and may lead to premature labor [18].

MRI is useful in characterizing uterine fibroids during pregnancy; subserosal fibroids can undergo torsion and degeneration during pregnancy. In our study MRI of 2 pregnant patients showed a subserosal uterine mass with signal intensity denoting a degenerating fibroid [1].

Most adnexal masses in pregnancy are asymptomatic and are discovered incidentally during ultrasonoraphy examination and most of it is non-neoplastic cysts [2].

In our study 3 cases of simple cysts, the MRI excluded the diagnosis of torsion as the ovarian architecture is preserved and no wall thickening and no surrounding edema, so reaching such diagnosis of uncomplicated ovarian mass, make the conservative treatment till delivery and the cyst was treated post partum by lapascopic cystectomy. So MRI finding that excluded the diagnosis of torsion saved the patient from unnecessary intervention surgery during pregnancy which would have been laparotomy with very high risk of preterm labor and its complication to the mother and fetus Adnexal torsion is the rotation of at least one turn of the ovaries, adnexa or the fallopian tube around the line of the tubo-ovarian ligament and the infundibulopelvic ligament [19].

Symptoms of ovarian torsion are often nonspecific, making it difficult to differentiate from other causes of acute abdominal pain. The classic presentation includes sharp, localized right or left lower abdominal pain and tenderness with a palpable abdominal mass and peritoneal signs. Waves of nausea and vomiting as well as pyrexia have been observed. In some cases, patients experience intermittent pain, making the diagnosis even more challenging [20].

Torsion must also be considered in pregnant patients presented with sever acute pain because enlargement of the uterus causes a shift in the position of adnexal structures that may then become torsed. The MRI appearance of ovarian torsion MRI appearance is enlarged and edematous solid ovarian tissue [21]. In our 2 cases with ovarian torsion this appearance was observed.

Inflammatory bowel disease, including Crohn's disease and ulcerative colitis, is also a diagnostic challenge during pregnancy, because many symptoms typical of pregnancy may also be signs of active inflammatory bowel disease.

MRI findings such as bowel wall thickening and mucosal enhancement can be seen in inflammatory bowel disease. Mucosal biopsy is needed to confirm the diagnosis of Crohn's disease or ulcerative colitis [1].

The limitations of our study include the small study population. Also multiple pathologies included in the study prohibited against obtaining significant results.

\section{Conclusion}

Our study shows that MRI can be used to identify a wide variety of abdominal and pelvic disease processes in pregnant patients 
Habash et al. Medical Imaging and Radiology 2013,

with acute abdominal or pelvic pain as it is an efficacious means of diagnosing acute appendicitis, ovarian torsions, and other adnexal diseases in the acute setting.

\section{Competing interests}

The authors declare that they have no competing interests.

Authors' contributions

\begin{tabular}{|l|c|c|}
\hline Authors' contributions & YHH & HSD \\
\hline Research concept and design & $\checkmark$ & $\checkmark$ \\
\hline Collection and/or assembly of data & $\checkmark$ & $\checkmark$ \\
\hline Data analysis and interpretation & $\checkmark$ & $\checkmark$ \\
\hline Writing the article & $\checkmark$ & $\checkmark$ \\
\hline Critical revision of the article & $\checkmark$ & $\checkmark$ \\
\hline Final approval of article & $\checkmark$ & $\checkmark$ \\
\hline Statistical analysis & $\checkmark$ & $\checkmark$ \\
\hline
\end{tabular}

\section{Acknowledgement}

The authors would like to thank their colleagues for the support and patients for their trust.

Publication history

Editor: Andrea Biscardi, Maggiore Hospital, Italy.

Received: 08-Nov-2013 Accepted: 04-Dec-2013

Published:19-Dec-2013

\section{References}

1. Birchard KR, Brown MA, Hyslop WB, Firat Z and Semelka RC. MRI of acute abdominal and pelvic pain in pregnant patients. AJR Am J Roentgenol. 2005; 184:452-8. | Article | PubMed

2. Cappell MS and Friedel D. Abdominal pain during pregnancy. Gastroenterol Clin North Am. 2003; 32:1-58. | Article | PubMed

3. Brown MA, Birchard KR and Semelka RC. Magnetic resonance evaluation of pregnant patients with acute abdominal pain. Semin Ultrasound CT MR. 2005; 26:206-11. | Article | PubMed

4. Valentini AL, Gui B, Basilico R, Di Molfetta IV, Micco M and Bonomo L. Magnetic resonance imaging in women with pelvic pain from gynaecological causes: a pictorial review. Radiol Med. 2012; 117:575-92. | Article | PubMed

5. ACR Practice Guideline for the Performance of Magnetic Resonance Imaging (MRI) of the Soft Tissue Components of the Pelvis. Reston, VA: American College of Radiology. 2006 (Amended). | Article

6. Schwartz LB, Panageas E, Lange R, Rizzo J, Comite F and McCarthy S. Female pelvis: impact of MR imaging on treatment decisions and net cost analysis. Radiology. 1994; 192:55-60. | Article | PubMed

7. ACR Practice Guideline for the Performance of Magnetic Resonance Imaging (MRI) of the Soft Tissue Components of the Pelvis. Reston, VA: American College of Radiolog. 2006(Revised). I Pdf

8. Roche O, Chavan N, Aquilina J and Rockall A. Radiological appearances of gynaecological emergencies. Insights Imaging. 2012; 3:265-75. | Article | PubMed Abstract | PubMed Full Text

9. Prokesch RW, Schima W, Berlakovich G and Zacherl J. Adrenal hemorrhage after orthotopic liver transplantation: MR appearance. Eur Radiol. 2001; 11:2484-7. | Article | PubMed

10. Chung JJ, Semelka RC, Martin DR and Marcos HB. Colon diseases: MR evaluation using combined T2-weighted single-shot echo train spinecho and gadolinium-enhanced spoiled gradient-echo sequences. $J$ Magn Reson Imaging. 2000; 12:297-305. | Article | PubMed

11. Low RN, Sebrechts CP, Politoske DA, Bennett MT, Flores S, Snyder RJ and Pressman JH. Crohn disease with endoscopic correlation: single-shot fast spin-echo and gadolinium-enhanced fat-suppressed spoiled gradi- ent-echo MR imaging. Radiology. 2002; 222:652-60. | Article | PubMed

12. Katzberg RW, Buonocore MH, Ivanovic M, Pellot-Barakat C, Ryan JM, Whang $\mathrm{K}$, Brock JM and Jones CD. Functional, dynamic, and anatomic MR urography: feasibility and preliminary findings. Acad Radiol. 2001; 8:1083-99. | Article | PubMed

13. Nagayama $M$, Watanabe $Y$, Okumura A, Amoh Y, Nakashita S and Dodo Y. Fast MR imaging in obstetrics. Radiographics. 2002; 22:563-80; discussion 580-2. | Article | PubMed

14. Mourad J, Elliott JP, Erickson L and Lisboa L. Appendicitis in pregnancy: new information that contradicts long-held clinical beliefs. Am J Obstet Gynecol. 2000; 182:1027-9. | Article | PubMed

15. Puskar D, Balagovic I, Filipovic A, Knezovic N, Kopjar M, Huis M and Gilja I. Symptomatic physiologic hydronephrosis in pregnancy: incidence, complications and treatment. Eur Urol. 2001; 39:260-3. | PubMed

16. Pazzi P, Gamberini S, Buldrini $P$ and Gullini S. Biliary sludge: the sluggish gallbladder. Dig Liver Dis. 2003; 35 Suppl 3:S39-45. | Article | PubMed

17. Singh AK, Desai $H$ and Novelline RA. Emergency MRI of acute pelvic pain: MR protocol with no oral contrast. Emerg Radiol. 2009; 16:133-41. | Article | PubMed

18. Coronado GD, Marshall LM and Schwartz SM. Complications in pregnancy, labor, and delivery with uterine leiomyomas: a population-based study. Obstet Gynecol. 2000; 95:764-9. | Article | PubMed

19. Huchon $C$ and Fauconnier A. Adnexal torsion: a literature review. Eur J Obstet Gynecol Reprod Biol. 2010; 150:8-12. | Article | PubMed

20. Chang HC, Bhatt $S$ and Dogra VS. Pearls and pitfalls in diagnosis of ovarian torsion. Radiographics. 2008; 28:1355-68. | Article | PubMed

21. Rha SE, Byun JY, Jung SE, Jung JI, Choi BG, Kim BS, Kim H and Lee JM. CT and MR imaging features of adnexal torsion. Radiographics. 2002; 22:283-94. | Article | PubMed

\section{Citation:}

Habash YH and Darwish HS. Magnetic resonance evaluation of acute abdominal and pelvic pain in pregnancy. Med Imaging Radiol. 2013; 1:2. http://dx.doi.org/10.7243/2054-1945-1-2 